\title{
Étude des caractéristiques géotechniques et d'érodabilité des sols de la ville de Manaus (Brésil)
}

\section{GOUTTE-LIMA}

SEMEN TP

La Barrière Noire, BP 14 44220 Couëron marisjg@hotmail.fr

J. CAMAPUM

DE CARVALHO

Université de Brasilia

Faculte de Technologie Programme de géotechnique

70910-900 Brasilia

$D F-B r e ́ s i]$

camapum@unb.br

\section{ALVES DA FROTA}

Université d'Amazonas

Faculté de Technologie Laboratoire de Mécanique des Sols

69073-970 Maraus

AM - Brésil

cafrota@yahoo.com.br
Cet article pre̊sente des relations entre les paramètres géotechniques obtenus pour les sols de la ville de Manaus, située dans la région amazonienne, au Brésjl. Cette recherche a pour objectif l'interpretation de l'érodabilitè des sols de quatre ravins dans la zone urbaine. Les résultats montrent que la minéralogie et les propriétés phiysiques de ces sols sont variables par rapport à la cote altimétrique de la ville. En effet, dans les cotes supérieures, le sol est argiteux, dans les cotes intermédiaires, il est argilo-sableux et dans les cotes plus basses, il devient sableux. Pour cela, nous avons fait des essais de comportement mécanìque en sols non remaniés d'un ravin situé dans une cote intermédiaire. Ces essais ont consisté en la mesure d'érodabilité dans l'appareil d'Inderbitzen, en cisaillement direct. en courbes de rétention d'eau et un essai SPT in situ. Des corrélations entre quelques caractéristiques physiques ont étế possibles. Pourtant, en fonction des particularités de structure et de la composition minéralogique de ces sols, les corrélations entre le comportement mécanique et l'érodabilité n'étaient pas si évidentes. Malgré cela, on remarque la forte influence de la nature du sol sur les caractéristiques géomécaniques et d'érodabilité du profil de sol d'un de ces ravins.

Mots-clés : corrélations, érodabilitté, gêotechnique, sols tropicaux.

\section{Study of the geotechnical characterization and soils erodibility in the town of Manaus (Brazil)}

\section{This paper presents of the relations between the soils} geotechnical properties obtained from the gullies in the town of Manaus, in the Amazonjan recion, in Brazil. This research aims at the interpretation of the soil erodiladity came from four gullies in the urban district. The results show that the mineralogy and the physical soils properties are variable with the level dimension of the city. Indeed, it was observed that the soil profile of the city is very uniform, with a shallow clayey layer in regions above the sea water level and a sand laver in regions below it. For that, we carried out tests of mechanical belavior for the undisturbed soils from of the gully irs the medium level. These laboratory tests consisted of the measurements of the erodibility soils from the Inderbitzen Test. Direct Shear, the curves characteristics and the field test SPT. The correlations between the soils properties were possible. However, according to the particularities of structure and mineralogical soil composition, the correlation between the other properties can's be obvious. In spite of that, it was noted the influence nature of the soil in the geomechanicals characteristics and erodibility in the soil profile of one of these gullies.

Key words : correlations, erodibility geotechnical, tropical soils. 


\section{Introduction}

La ville de Manaus, capitale de l’Etat d"Amazonas, située dans la région nord du Brësil, a une superficie de $11458,8 \mathrm{~km}^{2}$, sachant que l'État d'Amazonas a une superficie de $1558987 \mathrm{~km}^{2}$. Sa localisation se trouve entre les parallëles $03^{\circ} 00^{\circ} / 03^{\circ} 10^{\prime}$ S et les méridiens $59^{\circ}$ $55^{\prime} / 60^{\circ} 07^{\prime} \mathrm{W}$, au centre géographique de 'Amazonle. La ville est limitée par la rive cauche de la confluence des rivières Negro et Solimōes, quj Foment ensemble le fleuve Amazone.

La ville a un relier modéré dont l'altitude movenne est de $65 \mathrm{~m}$ au-dessus du niveau de la mer. D'après Fernandes Filho (1997), les surfaces topographiques les plus élevées de la ville de Manaus se trouvent près de la cote $100 \mathrm{~m}$ au-cessus du niveau de la mer. La région présente un climat hunicle et chaud, caractérisé paí deux saisons prédominantes : une saison des pluies (I"hiver\}, qui se prolonge du mois d'octobre au mois de juin et une saison sêche [l'été], du mois ce juillet au mois de septembre. Les précipitations annuelles movennes varient entre 2100 et $2500 \mathrm{~mm}$. ce qui provoque des crues périadiques dans la région.

D'aprés Fernandes Filho at al. (1997) la géologie régionale est représentée par les sédiments Meso-Cénozoïques de la formation « Alter do Chão y et les dépôts quaternaires de la formation u Solimōes n. La formation Alter do Chäo est uß système géologique du Cré tacé. caractérisé par des sédiments rouges et faiblement consolidés. Elle est constituée, essentiellement par des arénites, des limonites et des argilites, qui forment lo substratum rochelux de la ville. Ĺarénite de cette formation est connue, régionalement comme arénite Manaus $y$, crui est la principale source de cailloux et de graviers pour les travaux publics dans la région.

Les sols de la ville de Manaus sont classifiés, pédoJogiquenent, comme ulatosols jaunes m. Ces sols ont une texture variable entre argileuse, sablo-argileuse ou argile-sableuse et encore, sableuse, ils sont fortement draines avec une coloration jaunissante el riches en minéraux de quartz et en minérałx argileux du type kaolinite.

Le processus d'érosion dans la ville est provoqué principalement par la concentration excessive des eaux pluviales et la déforestation près des lotissements qui, associés aux caractéristiques des sols, est à l'origine des ravinements. Ce phenonène se manifeste sum la couche la plus superficielle du profil de latosol jaune, provoguant d'abord des ruissellements et ensuite de grands ravins sur le terrain. Dans la plupart des cas, ces ravins arrivent à 18 n de profondeum. I es études régionales en cours permettront de mieux connaitre les nécanismes d'origine et d'évolution de ces ravins. Elles aideront a concevoir des projets géotechniques de contrôle d'érosion dans la région. Cet article présente l'étude géotechnique réalisée sur quatre ravins situés dans trois zones et sur des cotes altimétriques différertes dans la ville de Manaus. Ces cotes ont été obtenues à l"aide d"un GPS et de la carte hyposométrique de la région. Sur la figure 1. on peut noter que les ravins étudiés sont le a Canara nas », qui se situé șur lá cote altimétrique $100 \mathrm{~m}$, u Distrito in entre les cotes altimétriques $75 \mathrm{~m}-100 \mathrm{~m}$ et finale ment, deux ravins nommés a Tarumä 1 n et "Tarumä 2 n entre les cotes $50 \mathrm{~m}-75 \mathrm{~m}$.
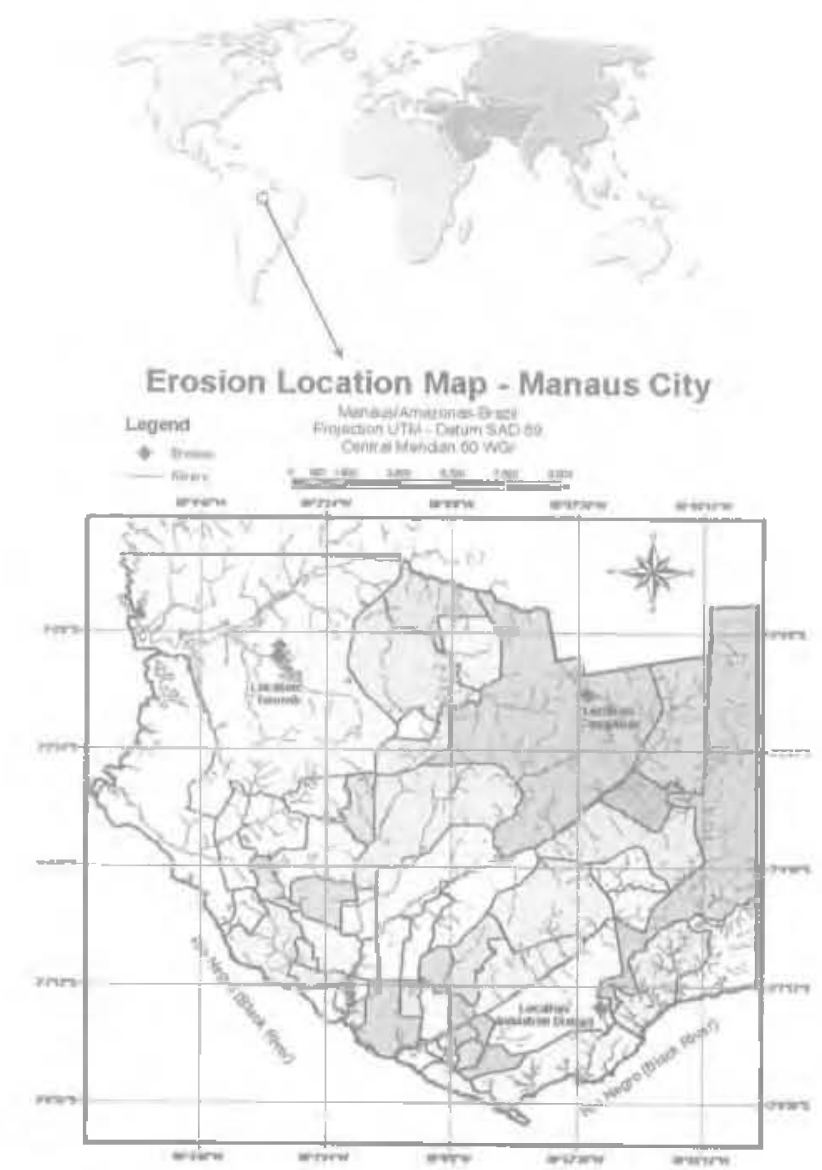

FIG. 1 Les mavins étudiés dans la zone urbaine de Manaus.

1] faut signaler aussi que sur la carte des nappes phréatiques présentée par Bento (1998), nous avons constate que sur les ravins Canaranas et Distrito, le niveau d'eau se trouve à des profondeurs supérieures à $30 \mathrm{~m}$. Cependant, dans les ravins Tarumà, la nappe phréatique se trouve à des profondeurs guj varient de 0 à $10 \mathrm{~m}$.

Cette recherche a nécessité un nombre considérable d’essals de caractếrisation physique et minéralogique sur les profils de sol de ces quatre ravins. Etant donné que le profil de sol du favin Distrito est situé sur la cote altimétrique intermédiaire, nous avons réa. lisé aussi des essais pour l'analyse du comportement mécarique et de l'érodabilité de ces sols. L'analyse des résultats est basée sur des corélations entre les paramètres géotechniques obtenus.

\section{2}

\section{La problématique d'érosion des sols au Brésil}

L'érosion ast définje comme l'ensemble des processus, par lesquels les matêriaux de l'écorce termestre sont détachés et transportés d'un endroit à l'autre par des agents érosifs, tels que l'eau (érosion hydrique), le vent (érosion éolienne) et la glace (érosion glaciaire). parmi d'autres. 
Lérosion hydrique, prépondérante au Brésil, est une conséquence du régime des plules, de l'action des vagues au bord des lacs, de la mer, de l'océan, maiss aussi tout au long des rivières et des fleuves. L'érosion par la pluie, qut est une des plus importantes dans les régions tropicales, a deux agents érosifs : la goutte qui tombe ef l'écoulement superfíciel qui en résulte.

Les régions du Brésil, comme les alentours de la capitale Brasilia, dans le District Fédéral et les villes de Golânia, de São Paulo, de Manaus et les Etats du Paraná et du Rio Grande do Sul sont beaucoup trop affectés par le problème d'érosion des sols. Cette situation est due a l'occupation désordonnée du sol datns les. communes urbaines et aussi par les pratiques parfois inappropriées clans les zones ruráles. Plusieur's recherches sont menées au Brésil afin de trouver des solutions aux problèmes géotechniques qui caractérisent les processus érosifs liés à l'érosion hydrigue.

Il faut remarquer qu'au Brésit, les régions affectèes par les processus de ravinement sont caractérisées par un profil de sol poreux, résiduelle ou non, où presque toujours la nappe phréatique est profonde. Dans ce cas, toutes les modifications physico-chimiques des sols sont provoqués par l'écoulement dans un milieu mon saturé, principalement après la formation des ravins due au processus d'érosion.

La formation du ravin peut aussi induire à un abałssement de la nappe phréatique, ce qui provoque le déclenchement de l'érosion interne et la dégradation physico-chimique du sol. par lessivage, près des talus de ces ravins.

Comme l'érosion dépend de nombreux tacteurs, son occurrence intervient de façon differente selor les régions, ce qui demande des études et des solutions particulières. Par exemple, dans le District fédéral fau Centre-Ouest du Brésil), la plupart des ravins ont une forme en " $V$ w dont la profondeur est limitée par les roches plus résistantes menant l'écoulement d'eau aै s'encaisser dans les couches moins résistantes du substratum rocheux (Mortari et Camapum de Carvalho, 1994). Selon Vilar et Prandi (1993). à São Paulo (dans le Sud-Est du Brésill) et au Paraná (clans le Sud du Brésil). les formatjons des ravins ont plutôt une forme en u U y et à Manaus (dans le Nord du Brésill], les ravins ont, en grande partie, une forme d'amphithétre [Lima, 1999]. La figure 2 montre ces types de ravins dans les villes de Manaus el de Brasilia. De cette manière, on peut constater que conme leurs formations et leurs évolutions sont différentes, il existe plusieurs propositions de modèles évolutifs pour explicuer ce processus érosif. Pour essayer de complendre le mécanisme d'évolution des érosions à Manaus, nous avons établi un programme d'essais de laboratoire et des observations de mécanismes de rupture sur te terrain.
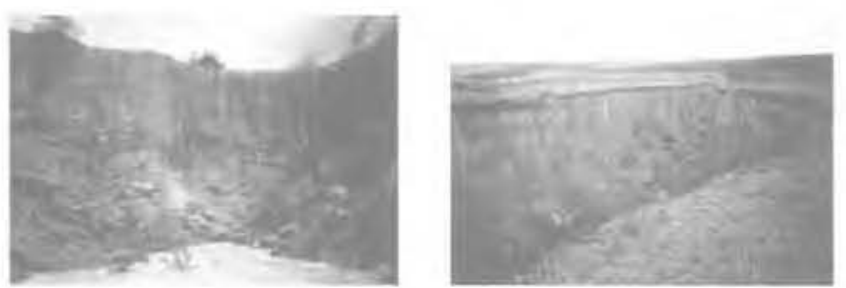

FG. 2 Ravins dans les villes de Manaus et District féctéral.

a) Ravin Distrito [Manaus! : bl Ravin District federal.

\section{Étude expérimentale}

L'étude expérimentale a été conduite sur quatre ravins représentatifs des érosions de la ville de Manaus nommés : Canaranas, Distrito. Tarumā 1 et Tarumā? (Fig. 1). Après la caractêrisation physico-minëralogique des profils de sols de chaque ravin, celui du Distrito a été choisi, en fonction de ses caractéristiques intermédiaires par rapport aux autres ravins, pour la poursulte des études sur le comportement mécanique et l'érodabilité des sols.

Les essais cle caractéristation physique ont consisté en la détermination de la teneur en eau naturelle ( $w$ ) du poids volumique des grains $\left(\gamma_{5}\right)$, des limites d'Atterberg ( $w$, et w) et des analyses granulonétrigues réalisées avec ou sans le défloculant.

La minéralogie a été déterminée en utilisant la technique de diffraction de rayons X sur les échantilons de sol passés sur le tamis de $74 \mu m$ (\# 200). Les James ont été confectionnées en exerçant une légère pression, pour éviter l'orientation de particules de sol.

Létude du comportement mécanique réalisée pour le ravin, situé sur la cote intermédiaire (ravin Distrito), a consisté en des essais cedométriques et de cisaillement direct des échantillons dans les conclitions saturées et non saturées, de déteminations de courbes de rétention d'eau et d'essais in situ, tels que le SPT (Standard Penetration Test) et SPT-T (mesure de la résistance a la torsion après chaque evaluation du nombre de coups dans l'essai SPT). Ainsi, l'essai SPT a étê réalisé sans circulation d'eau et avec des mesures de torsion en utilisant une clé dynamométrique avec aiguille suiveuse de capacité jusqu'à $48 \mathrm{Kgf}$.m. Les essais ont été réalisés tous les mè̀tes, avec des prélevèments d'échantillons, jusqu'à 13 m de profondeur. Les essais oedométriques réalisés dans les conditions saturée et non saturée ont permis d'évaluer le potentiel d'affaissement du sol pour les profondeurs de $3 \mathrm{~m}$ et de 5 m dù à la saturation. Les lésultats des essais en conditions saturées ont permis aussi de définir une vitesse de cisaillement dans la boite de Casagrande pour les essais drainés. Lessai de Casagrande dans les conditions saturée et non saturée a été réalisé pour les profondeurs de $1,3,5$, 7 et $8 \mathrm{~m}$. Il est possible, par exemple, de vérifier par l"inondation de l"échantillon, la susceptibilité des paramètres de résistance lorsque le sol a subi un événement pluvieux. Dans ce cas, la succion diminue significativement avec lä variation de la teneur en eau sur" le terrair.

Les courbes de rétention d'eau ont été mesurées par la technique du papier filtré. Les échantillons utilisés étajent ceux du carotlage lor's d"essai SPT et aussi des échantillons non remaniés prélevés aux profondeurs de $1,3,5,7$ et $8 \mathrm{~m}$.

L’érodabilité se définit par la facilité des particules des sols à être désagrégées et transportées, ce qui reflète la prédisposition d'un type de sol à résister aux efforts des agents érosifs. De cette manière, l'érodabilité est conditionnée essentiellement par la résistance du sol à l'action des agents érosifs. Les essais d'érodabilité réalisẻs ont été l'Inderbitzen Test (Inderbitzen, 1961], le Pinhole Test (Sherard ot ad., 1976) et des essais de désagrégation. On remarque ici que les données utilisées dans les corrélations avec les autres caractéristiques des sols, sont les pertes de sol obtenues dans l'essai d"Inderbitzen (conditions saturée et non saturée), car elles sont quantifiés, tandis que les autres (la désagrégation et le Pinhole Testl sont des nesures plus qualitatives. 


\section{Caractérisation physique et minéralogique}

Les tableaux I à IV montrent les résultats des caractéristiques physiques obtenus pour les quatre ravins. Ces résultats révèlent deux aspects importants pour les profils de sols étudiés. Le premier montre que la teneur en argile (déterminée avea l'agent dispersant) et les limites d'Atterberg ont tendance à diminuer par rapport à la profondeur dans chaque profil. Et le deuxième aspect montre, en observant tous les tableaux, que le sol du ravin Canaranas est plus argileux, ensuite que les sols du ravin Distrito possedent les caractéristiques d'une argile sableuse ou d"un sable argiteux et ceux des ravins Tarumā, sont les plus sableux. Comme chaque ravin se trouve dans une cote altimétrique différente, on peut aussi analyser ces propriétés par rapport a la cote altimétrique estimée pour chacun de ces ravin.

Dans les études de sols tropicaux, l'analyse minéralogique constitue un outil auxiliaire pour la compré- hension d'un profil d'altération de sol, des proprié. tés physiques et des comportements mécaniques des sols. Pour vérifier l’influence des minéraux argileux sur les propriétés physiques de ces sols, sur le comportement mécanique et sur l'érodabilité, on a considéré les valeurs de l'intensité des pics de la kaolinite et du quartz dans chaçue diffractogramme des rayons $\mathrm{X}$. La kaolinite est un silicate d"alumine qui résulte de l'altération et le quartz est un minéral frès résistant a la météorisation. La flöure 3 montre les profils des résultats de caractérisation physique et minéralogique pour les cutatre ravins en fonction de la cote altimétrique par rapport au niveau de la mer (Lima et al., 2000). Íensemble des résultats montre que la teneur en quartz et en kaolinite augmente avec la réduction de la cote altímétrique estimée pour les profils de sols. Cette figure met en évidence deux remarques : la continuité des profils des propriétés des sols avec la profondeur et l'êvidence d'un profil sédimentaire unique ; l'intempérisation due au climat est semblable daris les quatre profils des sols analysés et en accord avec la teneur en quartz, peu altérable.

unbisau Caractérisation physique (ravin Canaranas).

\begin{tabular}{|c|c|c|c|c|c|c|c|c|c|c|c|c|c|}
\hline \multirow{2}{*}{$\begin{array}{l}\text { Erot. } \\
\text { (m) }\end{array}$} & \multirow{2}{*}{$\begin{array}{l}w_{\text {nut }} \\
(\%)\end{array}$} & \multirow{2}{*}{$\begin{array}{l}W_{1} \\
(\%)\end{array}$} & \multirow{2}{*}{$\begin{array}{l}W_{\mu} \\
(\%)\end{array}$} & \multirow{2}{*}{$\begin{array}{l}I_{14} \\
{[\%]}\end{array}$} & \multirow{2}{*}{$\begin{array}{c}\gamma_{1} \\
\left(\mathrm{k} / \mathrm{V} / \mathrm{m}^{\mathrm{s}}\right)\end{array}$} & \multicolumn{4}{|c|}{ Avec dispersant $(\%)$} & \multicolumn{4}{|c|}{ Sans dispersanl (\%) } \\
\hline & & & & & & gravier & sable & limoty & argile & travier & stable & HกOI & arytle \\
\hline 1 & 37,0 & 74,4 & 41.4 & 33,1 & 20.3 & 0,0 & 13,0 & 11.5 & 75.5 & 0,0 & 76,0 & 56,0 & 28.0 \\
\hline 2 & 38,9 & 82.7 & 48.4 & 34,2 & - & - & - & - & - & & & - & - \\
\hline 3 & 39,6 & 84,5 & 49,9 & 34,6 & 20.0 & 0,0 & 9.0 & 13,5 & 77,5 & 0.0 & 9,0 & 64.0 & 27.0 \\
\hline 4 & 40,2 & 84,9 & 57.9 & 27,0 & & & - & - & - & - & & & \\
\hline 5 & 34,6 & 79.8 & 50,5 & 29,3 & $26, \pm$ & 13,5 & 11,5 & 17,0 & 60,0 & 11.0 & 11,0 & 46.0 & 32,0 \\
\hline 6 & 34.7 & 75,1 & 49.1 & 26,0 & & & - & - & - & - & - & - & $=$ \\
\hline 7 & 33,4 & 69,4 & 44.1 & 25,2 & - & & & & - & - & - & - & - \\
\hline 8 & 28,1 & 65,1 & 41.0 & 24,1 & 26,3 & 0,9 & 24,5 & 11,0 & 64,0 & 0,5 & 24,5 & 57,0 & $1 B, 0$ \\
\hline 9 & 26,8 & 57.9 & 38,3 & 19,8 & & & $=$ & - & $=$ & & - & - & - \\
\hline 10 & 24.6 & 53.7 & 33,2 & 20.5 & & & - & $=$ & $=$ & - & - & $=$ & - \\
\hline 11 & 24,9 & 43,0 & 29.1 & 13,9 & 26.3 & 0.5 & 41,5 & 12,0 & 46,0 & 0,5 & 40,5 & 32,0 & 27,0 \\
\hline 12 & 26,0 & 49.0 & 28.2 & 20,8 & - & - & - & - & $=$ & . & & " & - \\
\hline
\end{tabular}

TABLAU n Caractérisation physique (ravin Distrito).

\begin{tabular}{|c|c|c|c|c|c|c|c|c|c|c|c|c|c|}
\hline \multirow{2}{*}{$\begin{array}{l}\text { Prof. } \\
\text { [m] }\end{array}$} & \multirow{2}{*}{ Whot: } & \multirow{2}{*}{$\begin{array}{l}w_{L} \\
{[\%]}\end{array}$} & \multirow{2}{*}{$\begin{array}{l}W_{31} \\
\lfloor \% 1\end{array}$} & \multirow{2}{*}{$\begin{array}{c}1_{\mathrm{P}} \\
(\%)\end{array}$} & \multirow{2}{*}{$\begin{array}{c}\psi_{1} \\
{\left[\mathrm{kN} / \mathrm{m}^{3}\right]}\end{array}$} & \multicolumn{4}{|c|}{ Avec dispersant $[\%]$} & \multicolumn{4}{|c|}{ Sans dispersant ( $\%$ ) } \\
\hline & & & & & & graviet & sable & limon & argile & gravier & sable & limon & argle \\
\hline 1 & 25,2 & 61.5 & 40,0 & 21,5 & 26.40 & 25.0 & 38,5 & 10,5 & 26,0 & 25,0 & 30.0 & 19,0 & 6,0 \\
\hline 2 & 24,8 & 56,2 & 38.7 & 17,5 & & & - & $\Rightarrow$ & $=$ & - & - & - & - \\
\hline 3 & 17,1 & 46,3 & 30,1 & 16,2 & 26,26 & 1,0 & 51.0 & 10,0 & 28,0 & 1,0 & 57.0 & 290 & 13,0 \\
\hline 4 & 21,1 & 41,0 & 26.8 & 14,2 & - & $=$ & & & - & - & $=$ & $=$ & - \\
\hline 5 & 34,9 & 35,4 & 23,2 & 12,2 & 26.19 & 1,0 & 49.5 & 13,5 & 36,0 & 1,0 & 52,0 & 35,0 & 12,0 \\
\hline $\mathrm{B}$ & 15,3 & 34,6 & 21,5 & 13,1 & - & & - & - & - & - & & - & - \\
\hline 7 & 13,7 & 27,0 & 17,1 & 9.9 & 26,31 & 0,0 & 60.0 & 7.0 & 33,0 & 0,0 & 63.0 & 25.0 & 12,0 \\
\hline 8 & 10,4 & 22,8 & 17,6 & 5,1 & $=$ & - & - & - & - & - & - & - & - \\
\hline 9 & $14, \frac{4}{2}$ & 24,2 & 20,0 & 4,2 & 26,3 & 0,0 & 55 & 13 & 32 & 0,0 & 55 & 44 & 1 \\
\hline 10 & 11,1 & 24.6 & 19,1 & 5,6 & - & - & - & - & $=$ & - & - & - & - \\
\hline 11 & 6.3 & NL & $\mathrm{NP}^{3}$ & $\mathrm{NP}$ & 26.24 & 1,0 & 77,0 & 6,0 & 16,0 & 1,0 & 79,0 & 12,0 & 8.0 \\
\hline 12 & 11,1 & NL & $N P$ & NP & - & . & $=$ & $=$ & $=$ & - & & & \\
\hline
\end{tabular}




\begin{tabular}{|c|c|c|c|c|c|c|c|c|c|c|c|c|c|}
\hline \multirow{2}{*}{$\begin{array}{l}\text { Prof. } \\
\text { (nn3) }\end{array}$} & \multirow{2}{*}{$\begin{array}{l}w_{\min } \\
{[\%]}\end{array}$} & \multirow{2}{*}{$\begin{array}{l}W_{L} \\
|\%|\end{array}$} & \multirow{2}{*}{$\begin{array}{l}W_{p} \\
{[\%]}\end{array}$} & \multirow{2}{*}{$\begin{array}{c}1_{p} \\
(\%)\end{array}$} & \multirow{2}{*}{$\begin{array}{c}\gamma_{*} \\
\left(\mathrm{kN} / \mathrm{m}^{3}\right)\end{array}$} & \multicolumn{4}{|c|}{ Avec dispersant $(\%)$} & \multicolumn{4}{|c|}{ Sans dispersant $[\%]$} \\
\hline & & & & & & gravier & sable & !lmor & arghle & gravier & Sable & Limon & argle \\
\hline 1 & 9,2 & 18.9 & 14,8 & 4,1 & 26.6 & 3.0 & 71.0 & 9,0 & 17,0 & 1,0 & 74,0 & 21,0 & 4,0 \\
\hline 2 & 9,4 & 20,0 & 15,2 & 4,7 & & - & - & - & - & - & - & - & - \\
\hline 3 & 7,6 & NL & $\mathrm{NP}$ & $N P$ & 26.4 & 0,5 & 83,5 & 4,0 & 12,0 & 0.5 & 83,5 & $\$ 0,0$ & 6,0 \\
\hline 4 & 11,1 & 25,4 & 15.7 & 9,7 & & & & & & & & & \\
\hline 5 & $11_{r} 2$ & NL & $\mathrm{NP}$ & NP & & & & & & & & & - \\
\hline 6 & 15,6 & $\mathrm{NE}$ & $\mathrm{NP}$ & $N P$ & 26,5 & 0,0 & 87,0 & 3,0 & 10,0 & 0,0 & 92,0 & 7,0 & 1,0 \\
\hline 7 & 18,8 & NL & $\mathrm{NP}$ & $\mathrm{NP}$ & & & & & & & & & - \\
\hline
\end{tabular}

TAGLEA iv Caractérisation physique (ravin Tammā 2).

\begin{tabular}{|c|c|c|c|c|c|c|c|c|c|c|c|c|c|}
\hline \multirow{2}{*}{$\begin{array}{l}\text { Prot. } \\
\text { (m) }\end{array}$} & \multirow{2}{*}{$\begin{array}{l}w_{n o s} \\
\{\%]\end{array}$} & \multirow{2}{*}{$\begin{array}{l}w_{L} \\
(\%]\end{array}$} & \multirow{2}{*}{$\begin{array}{l}w_{p} \\
\mid \%)\end{array}$} & \multirow{2}{*}{$\begin{array}{l}I_{p} \\
(\%)\end{array}$} & \multirow{2}{*}{$\begin{array}{c}\gamma_{5} \\
\left(\mathrm{kN} / \mathrm{m}^{3}\right)\end{array}$} & \multicolumn{4}{|c|}{ Avec dispersant 1 \% $\mid$} & \multicolumn{4}{|c|}{ Stans dispersan: $\mid \% 1$} \\
\hline & & & & & & gravier & sable & Iimon & argile & gravier & sable & limon & argile \\
\hline 1 & 13.6 & 25,1 & 17.7 & 7,4 & 26.2 & 1,0 & 62,0 & 9.0 & 28,0 & 1,0 & 61,0 & 190 & 19,0 \\
\hline 2 & 8,6 & 17,6 & NP & $\mathrm{NP}$ & $=$ & - & - & $=$ & $=$ & " & - & $=$ & - \\
\hline 3 & 7,9 & NL & $\mathrm{NP}^{\mathrm{F}}$ & $\mathrm{NP}$ & 26,3 & 2,0 & 74,0 & 7,0 & 17,0 & 2,0 & 78,0 & 19,0 & 3,0 \\
\hline 4 & 11,3 & 19.2 & 15,1 & 4,1 & & & - & & & - & - & & - \\
\hline 5 & $\mathrm{~B}, 1$ & NL & $N P$ & $\mathrm{NP}$ & 20,2 & 1,0 & 81,0 & 3,0 & 15,0 & 1,0 & 83,0 & 10,0 & 6,0 \\
\hline 6 & 10.9 & 17.8 & 15.9 & 1.9 & & - & & - & - & - & - & & - \\
\hline 7 & 16,2 & 20,3 & 16.6 & 3.7 & & & & $=$ & & - & - & - & - \\
\hline 8 & 22.6 & 16,8 & 15,4 & 1,5 & 26,9 & 0,0 & 73.0 & 8,0 & 19,0 & 0,0 & 74,0 & 17,0 & 15,0 \\
\hline
\end{tabular}
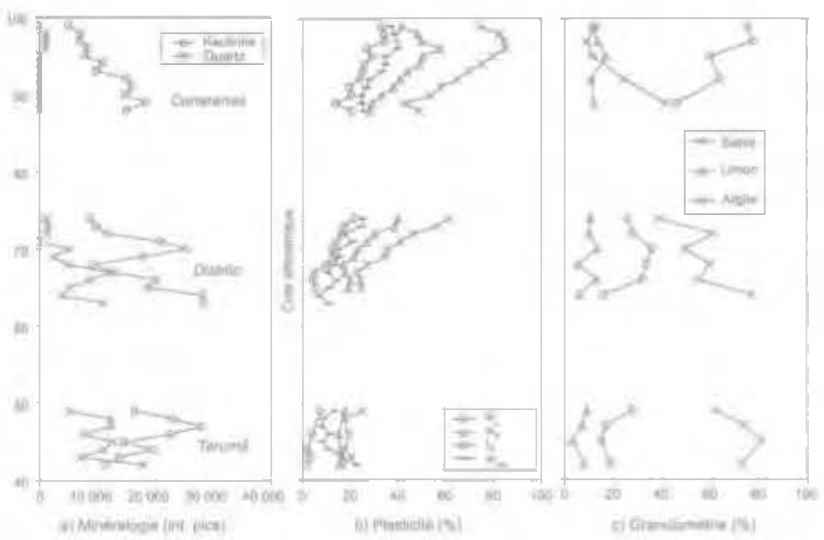

FG. 3

Essais de caractérisation géotechnique en fonction de la cote altimétrique.
On remarque d'une manière générale, dans tous les ravins, que la teneur en argile diminue par rapport à la profondeur et gue le pourcentage de limon est plus important dans les analyses granulométriques sans l'utilisation de dispersant, ce qui indique, qu'il s'agit, er partie, d'agrégats des sols et non seulement de particules indépendantes. La figure 4 montre le diagramme de Casagrande et la position de chaque ravin dans ce graphique. On peut constater que les sols du ravin Canaranas sont caractérisés comme $\mathrm{L}_{1}$, ceux du ravin Distrito, la moitié est $L$ et l'autre partie en fonction de la profondeur est $\mathrm{A}_{\mathrm{b}}$ et les sols du Tarumă sont classifiés comme $L_{i}$. On remarque dans ce diagramme que la majorité des points se trouvent au-dessous de la ligne $\mathrm{A}$, caractéristique non rare dans les sols tropicaux.

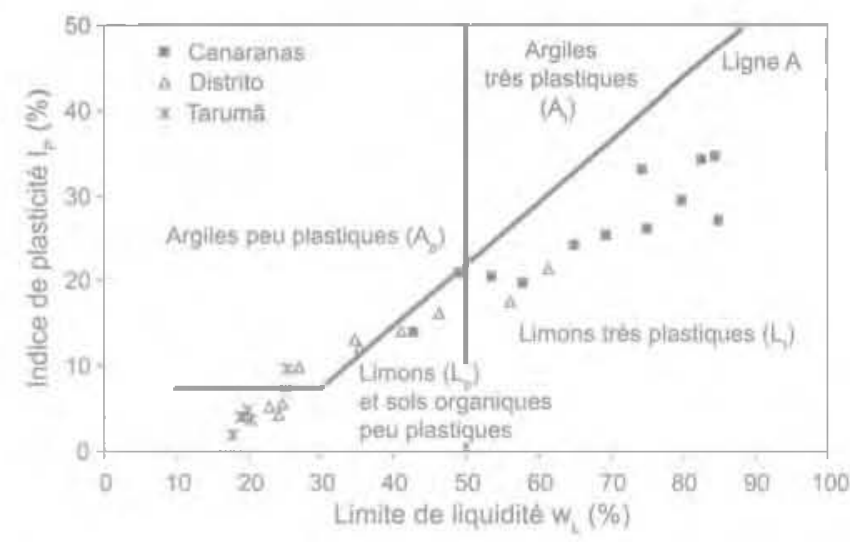

FIG 4 Diagramme de Casagrande.

\section{Comportement mécanique}

La figure 5 montre les profils de SPT (N), de torm sion (T) et de succion matricielle $\left(u_{3}-u_{w}\right)$ pour le ravin Distrito jusqu'a la profondeur de $13 \mathrm{~m}$. Dans ce cas les valeurs de succion ont été obtenues à partir déchantillons de sols prélevés lor des essais SPT. On remarque que les valeurs de $\mathrm{N}$ et des torsions (T') ont tendance à diminuer par rapport à l'augnentation de la profondeur jusqu'à $6 \mathrm{~m}$, et à partir de cette profondeur, ils ont tendance à augmenter. On peut observer aussi que les mesures de succion ont tendance à diminuer en fonction de la profondeur, ce qui est en accord avec 
les variations des propriétéc des sols, tels que la granulométrie, la plasticité et la teneur en eau naturelle tout au long de la profondeur. On peut dire aussi, que les paramètres $\mathrm{N}$ et $\mathrm{T}$ dépendent aussi bien de la succion que des propriétés physiques tel l'indice de vides.

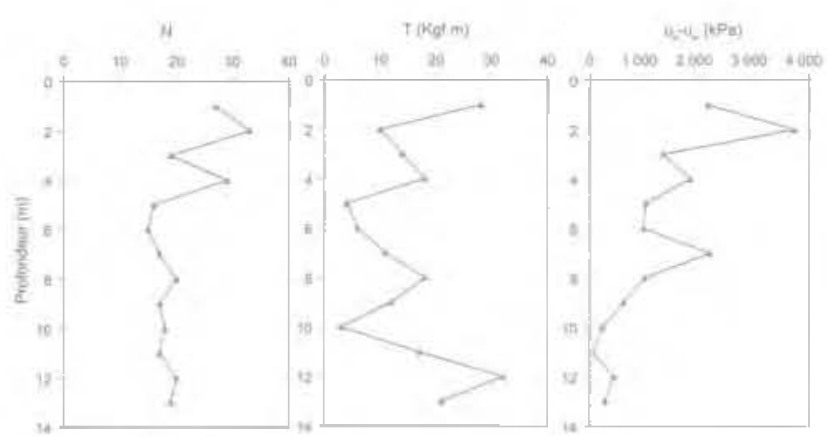

FG. 5 Profils de N (SPT), $T$ (torsion) et $\mathrm{u}_{\mathrm{a}}-\mathrm{UJ}_{\mathrm{w}}$ (succion) (ravin Distrito).

Le tableau V présente les paramètres de résistance ( $c$ et $\phi$ ) obtenus dans la boîte de Casagrande (cisaillement direct) pour les conditions naturelle (non saturée) et saturée (succion = zéro) à des profondeurs de 1,3 , 5,7 et $8 \mathrm{~m}$. Ces résultats montrent une réduction de la cohésion du sol avec l'inondation au fur et à mesure que la profondeur augmente.

TAlleauy Paramẻtres de résistance (ravin Distrito).

\begin{tabular}{|c|c|c|c|c|}
\hline \multirow[t]{2}{*}{ Profondeur (m) } & \multicolumn{2}{|c|}{$\begin{array}{c}\text { Naturel } \\
\text { (non saturé) }\end{array}$} & \multicolumn{2}{|c|}{$\begin{array}{l}\text { Inondé } \\
\text { (saturé) }\end{array}$} \\
\hline & c $(k \mathrm{~Pa})$ & $\infty\left[^{\circ}\right]$ & $\mathrm{c}(\mathrm{kPa})$ & $\phi\left\{{ }^{n}\right\}$ \\
\hline 1,0 & 64,8 & 39,5 & 28,8 & 38,5 \\
\hline 3,0 & 41 & 31,3 & $1 \mathrm{~B}$ & 31,4 \\
\hline 5,0 & 11,3 & 35,4 & 5,6 & 41.1 \\
\hline 7.0 & 24 & 35,7 & 3 & 32,2 \\
\hline 8,5 & 69 & 27,1 & 41 & 22,4 \\
\hline
\end{tabular}

\section{3}

\section{Essais d'érodabilité}

L'érodablité des sols a êté déterminée par l’essai d'Inderbitzen (1961). Cet essai consiste à passer un écoulement d'eau sur un échantillon de sol avec une déclivité de $10^{\circ}$ et un débit dé $50 \mathrm{ml} / \mathrm{s}$. Les valeurs de perte de sol sont déterminées après un temps d'essai de 30 minutes d'écoulement d'eau. Ces résultats sont montrés dans le tableau Vl. L'unité des valeurs de perte de sol est donnée par $\left(\mathrm{g} / \mathrm{mm}^{2}\right)$. Les essais ont été réalisés sur des échantillons naturel [non saturé) et saturée (succion = zéro), pour le ravin Distrito. Ces résultats montrent une liaison directe entre l'érodabilité (perte de soll et la cohéslon du sol saturé. Il est cependant important de remarquer que pour la condition non saturée, il n'y a pas une liaison directe entre la cohésion et l'érodabilité dî à l'influence contraire de la réduction de la succion lors de l'écoulement. En effet, lig pénétra. tion de l'eau dans le sol non saturé engendre, dans les pores, une pression d'air qui provoque le détachernent de particules quand elle dépasse la cohésion effective ou la cimentation du sol.

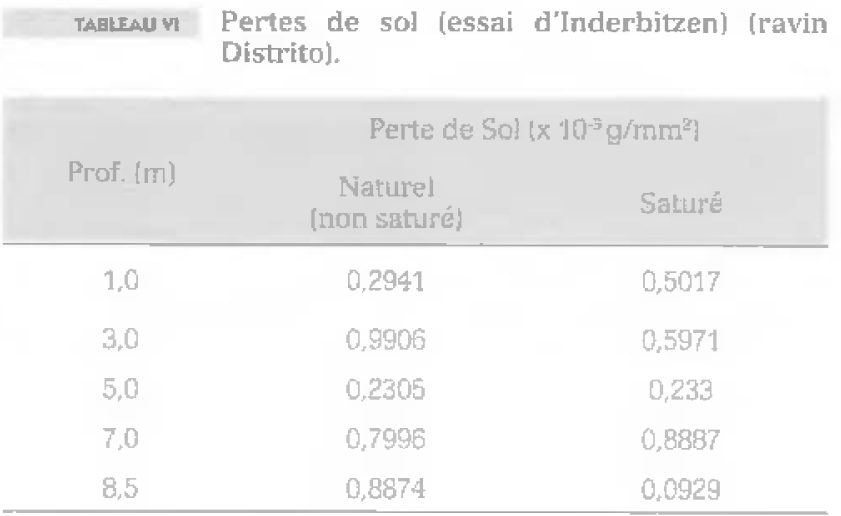

\section{4}

\section{Courbes de rétention d'eau}

Les mesures de succion ont été faites, par sêchage, en utilisant la technique du papier filtre, d'aprês les recommandations de l'ASTM (1992). Nous avons essayé de les mettre en corrélation avec les paramétres géotechniques obtenus et de vérifier l'influence directe de ceux-ci sur l'érodabilité des sols pour la région urbaine de Manaus. La figure 6 montre les courbes de rétention d’eau pour le profil de sol sur le revin Distrito. On remarcue les grandes variations de succion sur la couche superficieile a $1 \mathrm{~m}$ au-dessous du niveau du terrain. Il est évident que cette couche a été plus exposée à la météorisation, soumise à des cycles de séchage êt čhumidification

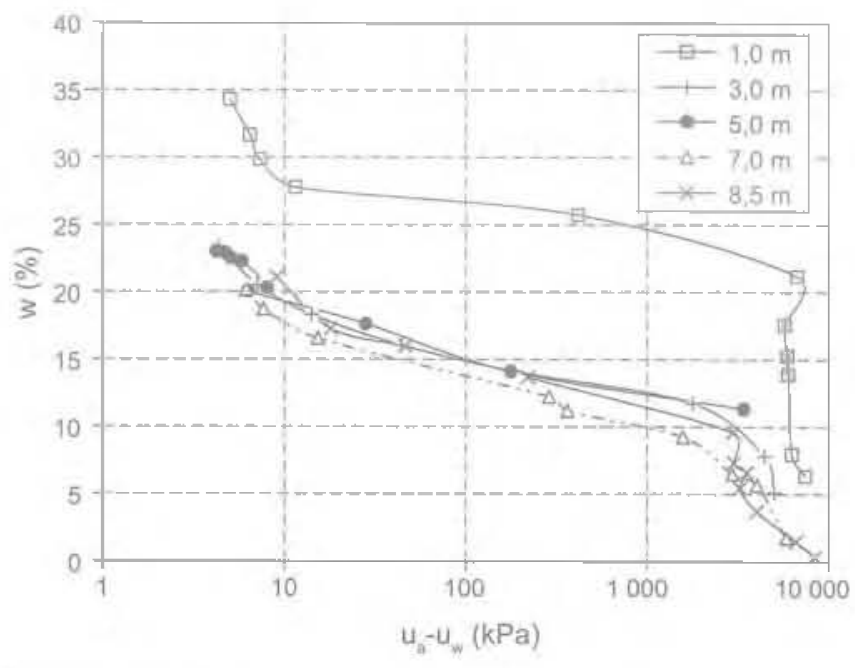

FG * Courbes de rétention d'eau (ravin Distrito). 


\section{Corrélations}

\section{1}

\section{Propriétés physiques}

Les corrélations présentées ici ont été ajustées aux meilleures tendances. Les résultats de caractérisation physique montrent que les sols du ravin Canaranas sont plus argileux, étant identifiés par une plasticité élevée et des valeurs importantes de la teneur en eau naturelle. Les sols du ravin Distrito sont caractérisés comme des sables argileux et movennement plasticues. Enfin, les sols du ravin Tarumā sont plus sableux par rapport aux deux autres profils analysés, présentant une faible plasticité, voire aucune plasticité dans les grandes profondeurs.

En fałsant la corrélation entre les résultats de la teneur en eau naturelle ( $w_{\text {nn }}$ ) et les propriétés de consistance ( $w_{1}, w_{p}$ et $I_{p}$ ) des profils des ravins analysés, on observe qu il existe une tendance à la corrélation linéaire entre ces indices, en montrant une augmentation de la teneur en eau par rapport aux propriétés plastiques (Fig. 7). On peut aussi vérifier sur cette figure que les caractéristiques d'humidité et de plasticité des profils des ravins se décalent selon la position des cotes altimétriques où ils se trouvent. Ce résultat révèle, en plus de la caractérisation du profil de sol typique pour la région de Manaus, que la composition minéralogigue de ces sols est fondamentalement lá même.

La figure 8 confirme l'influence du pourcentage d'argile sur les limites de plasticite ( $w_{1}$ et $w_{p}$ ) et l'indice de plasticité $\left(\mathrm{I}_{\mathrm{p}}\right)$, c'est-à-dire, plus le pourcentage d'argle est élevé, plus les limites de plasticité, les indices de plasticité et les teneurs en eau naturelles seront importantes. Il faut remarquer que l'inclinaison de la droite sur la figure 8 , qui corrèle l'indice de plasticité et le pourcentage d"ar= gile, représente le coeff́icient de l'activité moyenne de Skempton [I $\}$, qui est d'environ 0,46 , ce qui classifie ces sols comme inactifs. La plus grande dispersion de ces résultats par rapport aux résultats de la figure 7 peut être, au moins en partie, expliquée par le fait que, sur certe figure les résultats ont été obtenus pour les sols à l'état d'agrégation naturel, sur la figure 8 , la teneur en argile a été obtenue après la désagrégation du sol par une solution de céfloculant.

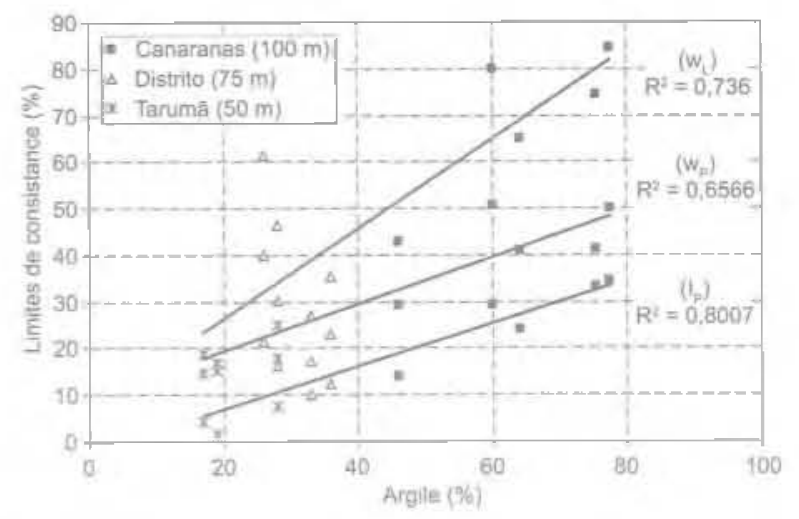

F1G. B

Corrélations entre le pourcentage d'argile et les linutes de consistance.

Benevelli et al. (2003) montrent gue dans les sols tropicaux, le niveau de désagrégation par laction de la solution défloculant dépend de la stabilité des agrégats et conséquemment du niveali de la météorisation qui a contribué pour la formation du sol.

Dans un profil de sol tropical, l'action des intempé* ríes est généralement minimisée en fonction de l'augmentation de la profondeur, indépendamment de la cote topographique du relief. En analysant ces mémes corrélations avec le pourcentage drargile sans l'utilisation de la solution défloculant, nous avons constaté la même tendance du graphique vu dans la fĭgure 8 .

Les coefficients de corrélation entre la plasticité et la somme des pourcentaces d'argile et de limon ('Fig. 9) ont été légèrement supérieurs aux coefficients, où on avait considéré seulement le pourcentage d'argile (Fig. 81. Les tableaux I à IV montrent que dans les analyses granulonétriques sans l'utilisation de la solution défloculant, le pourcentage de limon est trop élevé, ce qui peut indiquer que, soit la fraction limon est active, soit, elle est transformée en argile, dans les analyses avec l'utilisation de la solution défloculant.

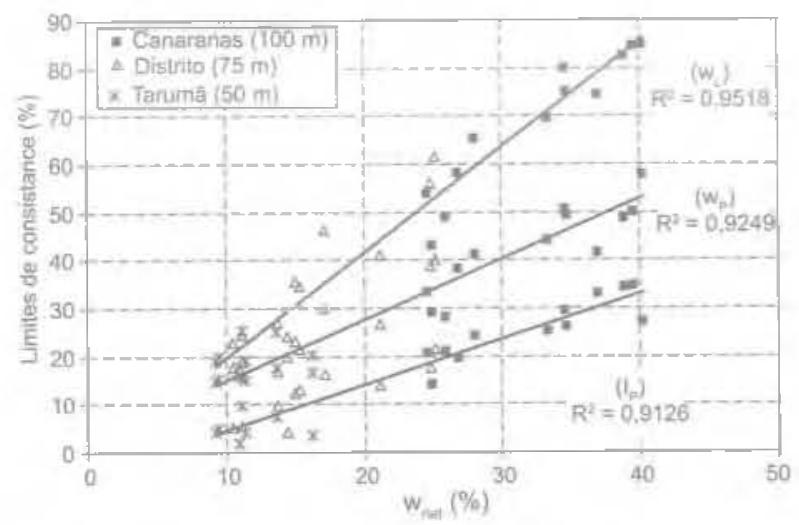

F16. 7 Corrélations entre $w_{\text {ar }}$ et les limites de consistance.

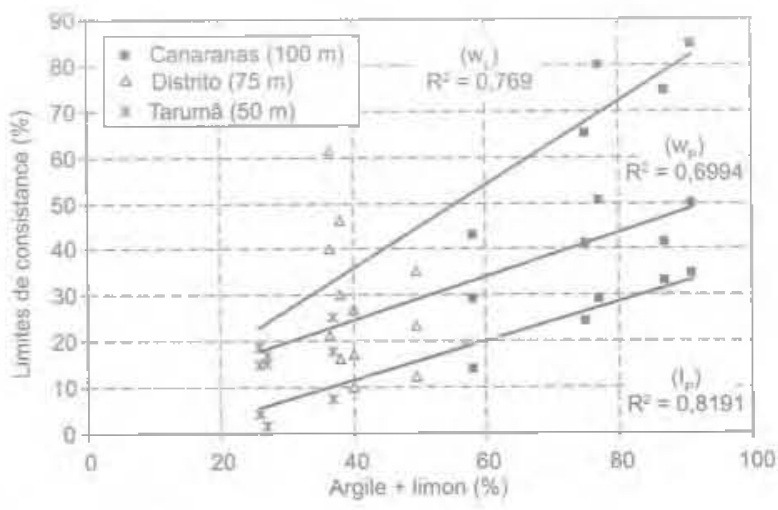

Fi6. Corrélations enfre les sommes d'argile et de limon et les limites de consistance. 


\section{Propriétés physiques et minéralogiques}

Afín d'analyser le niveau de comrélation existant entre la minéralogie, les limites de plasticité et la granulométrie, nous avons déterminé les intensités des pics de la kaolinite et du quartz. Ces valeurs ont été obtenues à partir des diffractogrammes des essais aux rayons $x$ z chaque profondeur.

La figure 10 présente le graphique de corrélation entre la kaolinite et l'indice de plasticité. Cette mône tendance a été observée pour les limites d'Atterberg. Cette figure montre qu'il y a une diminution de la un teneur n de la kaolinite avec l'augmentation des propriétés plastiques et conséquemment du pourcentage d'argile. Ceci paraít contrarier les concepts sur les limites de plasticite, dont les mesures sont dépendantes du type et de la quantité des minéraux argileux présents dans les sols.

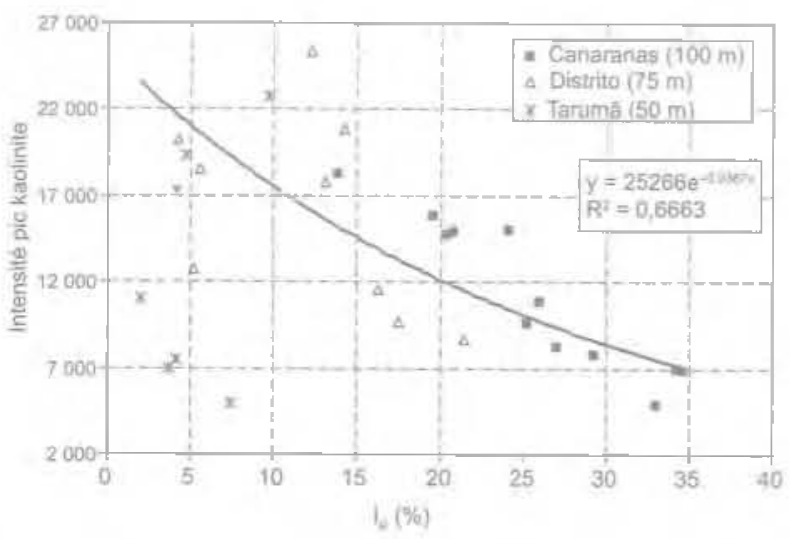

FIG. 10 Corrélation entre l'intensité du pic de la kaolinite et l'indice de plasticité.

Cette relation est due, probablement, à la présence d’un autre type de minéral argileux qui n'a pas pu être identifié dars les analyses de rayons $x$ et que sa présence a une influence remarquable sur la plasticité de ces sols. Cardoso (2002) montre que la plasticité des sols tropicaux augmente avec la teneur en oxyhydroxyde de fer. Il est possible qu'une analyse minéralogique complémentaire puisse nous expliquer ces corrélations inverses. Toutefois, dans cet ensembie, les dispersions entre les deux paramètres sont importantes et on remarque la nécessité de la compréhension des résultats à paltir des profils dintempérie et de la formation des sols dans la région.

On peut observer que sur les cotes supérjeures, et partant du domaine plus argileux (profil Canaranas et partie du profil Distritol. les propriétés de sols sont groupées dans une tendance prédéterminée et le reste dans une autre tendance, mais en gardant un certain parallélísme entre elles. Même si dans cet article, on ne présente pas la corrélation de l'intensité de pic de la kaolinite avec la teneur en argile, on remarque qu'elle a la mêne tendance que celle observée sur la figure 10.

Sur la figure 11, on trouve ta corrélation espérée existante entre l'intensité du pic de quartz et l'indice de plasticité. C'est-à-dire que plus importante est la teneur en quartz, plus petit sera l'I ${ }_{\text {. }}$ Ces résuitats confírment les hypothèses antérieures puiscu'il y a une honne corrélation entre ces deux paramètres. Or comme l"a!tération du quartz est difficile, il peut être considéré comme un élément tampon. La figure 12 montre une bonne relation entre le pourcentage de sable et l'intensité du pic de guartz, ce qui démontre que la fraction de sable est, en grande partie, quarzeuse.

Les corrêlations vues ci-dessus ont montré que les différences tout au long des profils, de sols de même qu'entre les ravins, sont fortement associées aux variations de cotes altimétriques. Chaque profil de sol étudié a été affecté par les processus d'intempérie et de météorisation qu'il a subis tout au long du temps. C'est-à-dire, que sur les cotes similaires, tous avons des propriétés et des caractéristiques des sols similaires. Cela pourra changer si le processus d"intempérie et la matière organique ont agi différemment.

Fatalement, c'est le cas poun" les cotes de ces ravins. ce qui interfère imperceptiblement sur les propriétés et le comportement mécanique de ces sols. If est évilent, cependant, que le ravin Distrito, en terme de caracté ristiques de sol, présente un comportement intemédiaire si on le compare aux autres ravins étudiés, ce qui nous a falt opter alors pour la poursuite et l"approfondissement des recherches sur ce ravin.

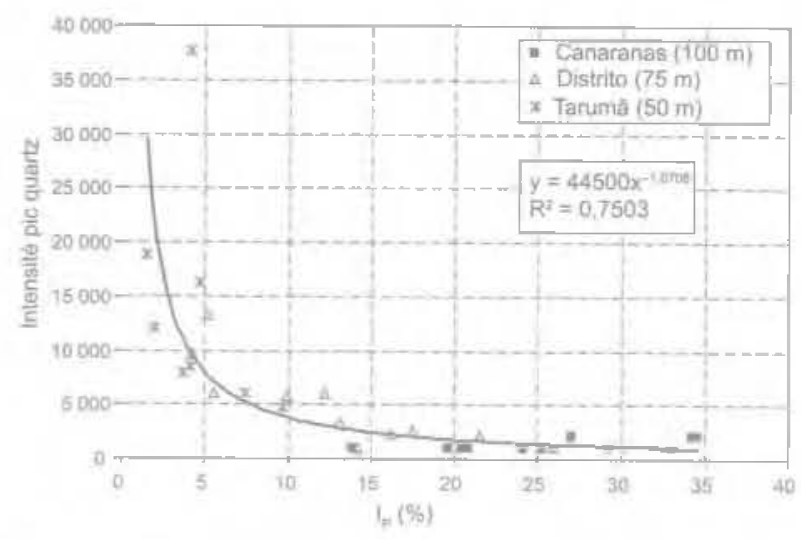

FIG. 11 Corrélation entre l'intensite du pic du quartz et l"indice de plasticité.

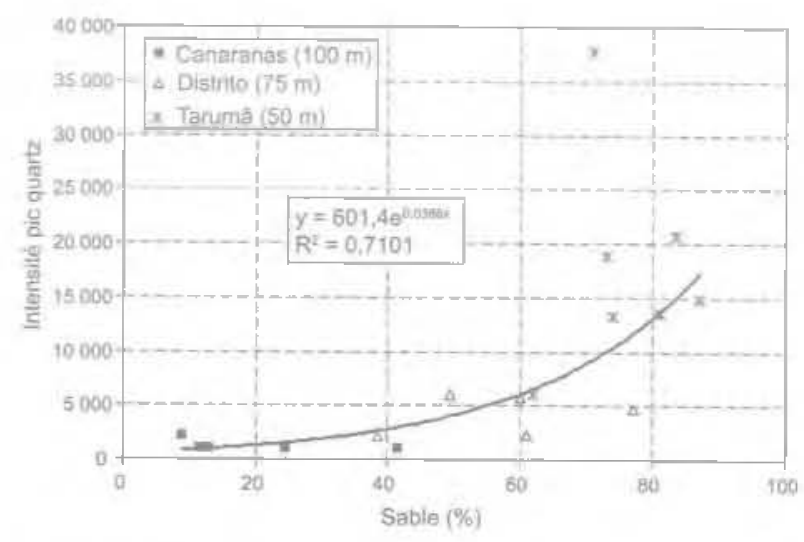

FIG. 18 Corrélation entre l'intensitté du pic du quartz et le pourcentage die sable. 


\section{Propriétés physiques, minéralogiques et de comportement mécanique}

Les analyses de corrélations entre les caractéristiques physico-minéralogiques et le comportement mécanique ont été obtenus pour le ravin Distrito. Il faut signaler que nous avons travaillé avec la tendance des graphiques car le nombre des résultats a été linnité.

Le graphique de la figure 13 met en relation les valeurs de cohésion et les intensités de pics de la kaolinite. Cette figure montre que plus l'intensite de pic de la kaolinite est inportante, plus la cohésion du sol est éle. vée, tandis qu'en faisant la mème relation pour l'angle de frottement, on a trouvé un comportement inverse, comme on pouvait l'espérer. Un autre aspect doit être mis en question sur ces résultats : la kaolinite n'est pas un minéral argileux très actif et pourtant il est cohésif. Il faut remarcuer que, dans le cadre de la météorisation des sols tropicaux, les oxyhydroxycles de fer et d'aluminium contribuent à un type de cimentation pour ces sols, en plus de la kaolinite. Leroueil et Barbosa (1999) introduisent la composante de cohésion due à la cimentation dans la résistance des sols tropicaux.

Nous n'avons pas pu obtení de relations entre les paramètres de résistance et les intensités de pics de quartz. D'après le graphicue de la figure 12, la phase granulaire de ce sol, à l'état naturel. peut être constituée d'autres minéraux, en plus de quartz, ce qui provoque la dispersion entre les points. Le même comportement a été observé pour des relations avec la somme entre l'argile et le limon.

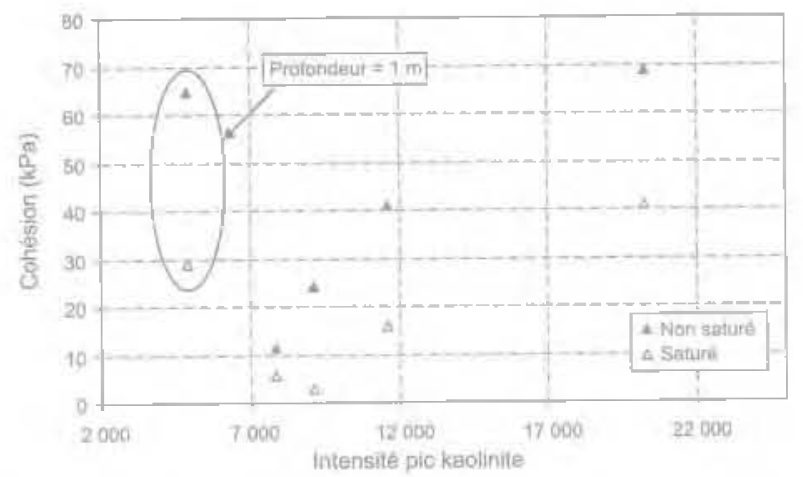

F1G. 13 Corrélation entre la cohésion et l'intensité du pic de la kaoliuite.

Dans les sols tropicaux, la fraction limon el sable est, cans la plupart des cas, composée par des agrégats d'argile, limon et/ou sable. Le ciment dans ces agrégats peut étre formé par ponts d'argile ou par oxyhydroxydes de fer et/ou d'aluminium.

Benevelli et al. (2003), montrent que les agrégats existants sur les profils des sols tropicaux ne sont pas, en leur majorité, désagrégeables sous l'effet d'hexamètaphosphate de sodium, qui est la solution de défloculant utilisée dans les essais de sédimentation au Brésil. Il faut remarculuer que certains agrégats présents dans les sols tropicaux demandent de l'énergie mécanique pour être défaits.
Nous savons que le tvpe de minéra! d'arqile, l'indice de vides et le degré de saturation ou la teneur en eau, sont des facteurs importants dans la definition de la succion d'un sol. En plusieurs cas, les corrélations avec chacun de ces facteurs séparément, conduisent à déterminer, parmi eux, lequel exercera une plus forte influence sut" la succion pour un certain type de sol. Dans l'étude de la succion matricielle du profil de sol du ravin Distrito, nous avons analysé l'influence de la nature du sol sur ce parametre. D'autres analyses sont présentées par Lima (1999).

Les mesures de succion utilisées dans la corrélation ont étéc obtenues a partir des échantillons prélevés avec l'humjdité nature!le pendant l'essai du SPT. Polr cela, on a utilisé la technique du papier filtre.

Comme la succion dêpend de ta composition granulométrique, celle-clétant d'autant plus importante que la sol est plus argileux, on a essayé d'établir ume corrélation entre les propriétés plastiques et les mesures de succion matricielle. La figure 14 montre la relation entre la succion matricielle et l'activité de Skempton (I $\mathrm{I}_{\mathrm{a}}$ ) pour ces sols, ce qui indique qu'il existe une influence de la nature du sol dans son comportement.

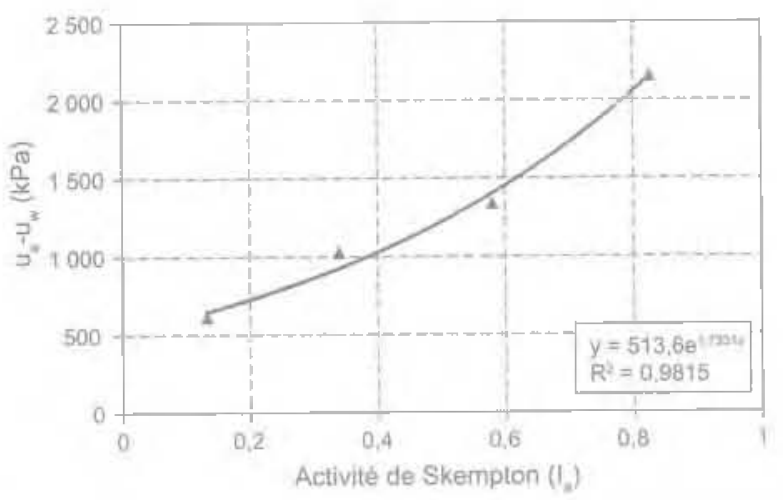

F1G. t4 Corrélation entre ta succion matricielle et l'activité de Skempton.

\section{Propriétés physico-minéralogiques, comportement mécanique et érodabilité}

La corrélation entre l'érodabilité des sols et les paramètres géotechniques est très difficile due au grand nombre de variables qui interferent dans cette propriété. Les essais d'érodabilité n'ont étê réalisés que pour le ravin. Distrito. Ainsi, nous avons obtenus quelques relations entre les valeurs de pertes de so] (P.S.) trouvees dans l'essaí d'Inderbitzen et les autres propriétés des sols.

Il faut tenir compte du fait que dans les essais d'Ir' derbitzen sur des sols non saturés, au fur et à mesure que les essais sont réalisés, le degré de saturation, et par conséquent, la succion et le comportement propre du sol vont changer. Ces altérations se produisent au cours d'essais imposant des limitations pour l'obtention de corrélations avec les autres propriétés du sol. La seule tendance de corrélation acceptable de la perte de sol à l'état initial non saturé a été obtenue avec la fraction de sable (Fig. 15). 


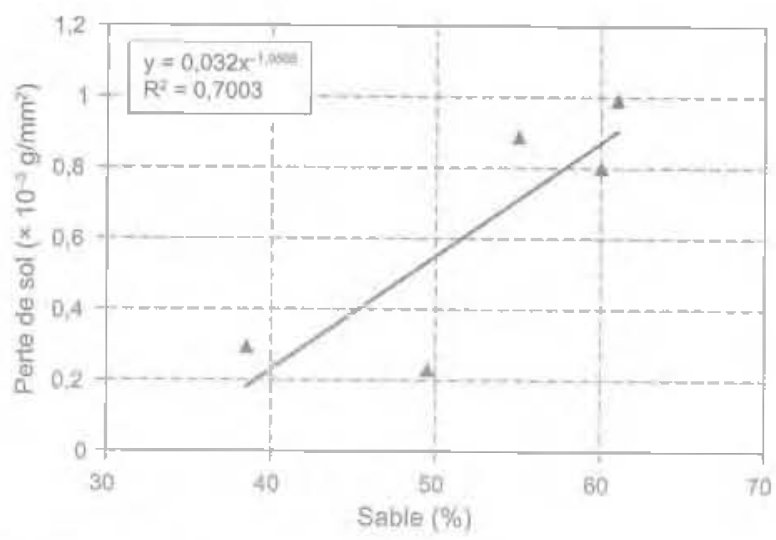

Fla. is Corrélation entre la perte de sol (non saturé) et le pourcentage de sable.

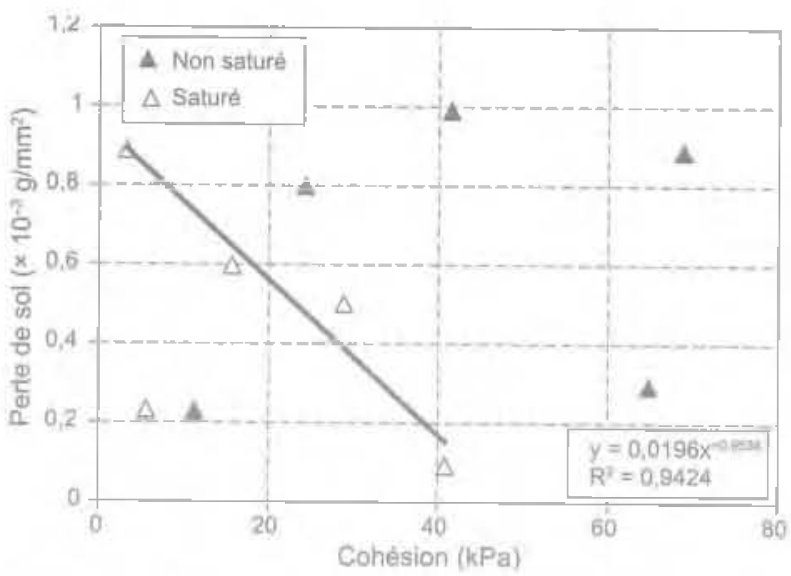

FIG 17 Corrélation entre la perte de sol et la cohésion.
La figure 16 montre la corrélation entre la perte de sol (échantillon à l'état saturé) et le pourcentage de limon obtenu sans l'utilisation de la solution défloculant. On peut noter l'influence positive du limon à l'état naturel dars l'érodabilite. On remarque que cette même tendance a été observée pour la relation avec de la kaolinite. Dans la condition saturée, i] est possible de veriffer que la perte de sol, et donc l'érodabilité, augmente avec la diminution de la cohésion du sol. alors que dans la condition non saturée, on n'a trouvé aucune tendance (Fig. 17). La figure 18 montre cependant qưil y a une bonne corrélation entre la perte de sol, à l'état initial saturé et la variation de cohésion $(\Delta c)$. Dans cet article, la variation de cohésion $(\Delta c)$ est donnée par l'écuation suivante:

$$
\Delta c=\frac{C_{\text {Requre }}-C_{\text {nansature }}}{C_{\text {nonsalure }}} \times 100
$$

Dans les sols tropicaux, Bender ( 1985 ) a vérifié que l’érodabilité est directement liée à la résistance au cisaillement. Ce fait a été constaté aussi par Bastos (1999). Dans cet article, pour les sols de Manaus, on a remarqué quril y a aussi une relation entre la variation de la résistance au cisaillement et les valeurs d'érodabilité (perte de sol saturé).

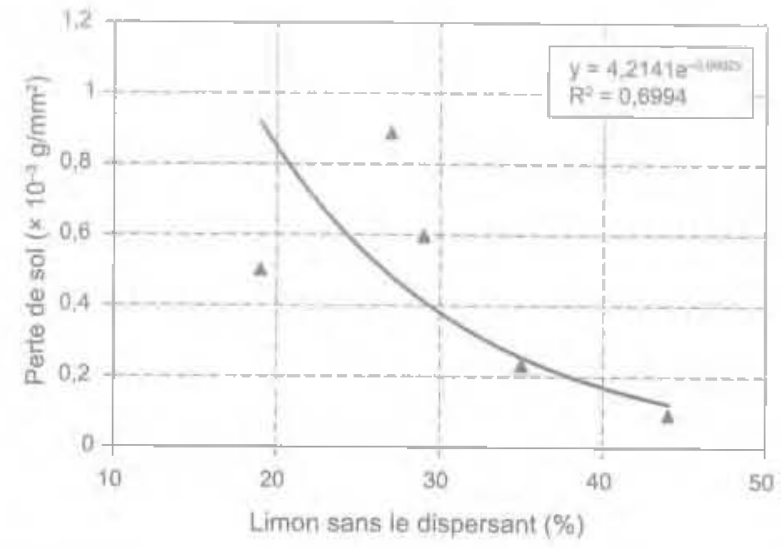

FiG. I6 Corrélation entre la perte de sol et le pourcentage de limon sans le dispersant.

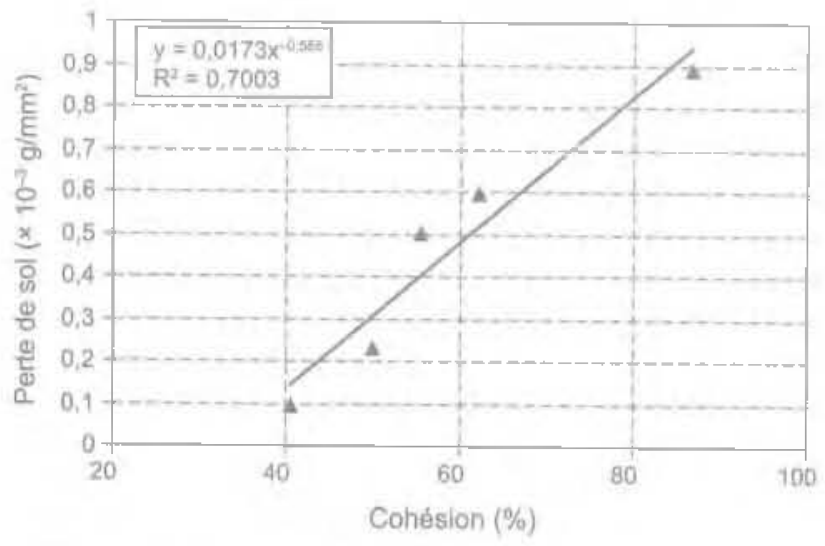

Fit. 18 Corrélation entre la perte de sol et la variation de perte de cohésion.

\section{5}

\section{Conclusion}

Des résultats, on peut constater que :

- les caractéristiques de la teneur en eau, de la plasticité et les fractions granulométriques des ravins étudiés dans la ville de Manaus se trouvent alignés et ne sont décalés que par les positions sur les cotes altimétriques;

- les relations entre les pourcentages d'argile, les limi tes de plasticité et lá teneur en eau sont convenables et cohérentes. Dans ce cas, les résultats valident l'influence de l'argile sur les propriétés plastiques du sol et sur la teneur en eau naturelle, ce qui revient à dire que, plus le pourcentage d'argile est important, plus les limites de liquidité et de plasticité, l'indice de plasticité et aussí la succion sont élevés;

- les caractéristiques de succion, les paramètres de cohésion et aussi l'érodabilité sont influencés par la nature du sol : 
- la perte de cohésion de sol due au processus de satu ration montre un excellent indicateur pour la prévision de l'érodabilité pour les sols tropicaux:

- les meilleures relations ont été obtenues avec les valeurs de perte de sol dans l'état saturé. Étant donné les facteurs qui influencent conjointement les propriétés des sols dans l'état naturel tels que : la succion, la cimentation, la macrostmacture doriginc pedologigud ou géologique, les relations avec la perte de sol à l'état naturel n'ont pas été possibles :

- en fonction du grand nombre de variables intervenants dans l'érodabilité des sols, leurs relations avec d'autres propriétés des sols présentent des difficultês non négligeables.

\section{$\overline{\text { Bibliographie }}$}

ASTM 1997. D-5298 - Standard test method for measurement of soil potential (Suction) tring fitter paper. Anintal Book of ASTM Standards, vol. 15.09 , p. $1312-1316$

Basto C.A.B. - Estudo Geotécnico sobre a erodibilidade de solos residuais na sath. rados. Thè̀se de dociorat en génie civil, UFRGS, Porto Alegre, RGS, Brésil. 1999. $270 \mathrm{p}$

Bender H. - Érosion : un problême de résistance au cisaillement en fonction du chemir des constraintes pendant l'infiltration. international Conference on Geomechanics in Tronical Lateritic and Saprolitic Soils. Proceedings, Brasilla/DF, Brésil, ABMS, 1985, vol. 2, p. 15-25.

Beneveli R.M. Oliveira C.G.M. Camapurn de Carvatho J. - Influéncia do tipo de defloculante de particulas finas na determinaçăo da granulometria, ISympostum de Géotechnique du Centre-Ouest. Brasilia, DE, Brésil. 2003 (document rumérique).

Bento A.H. - Mapeamento Geotercieg de Aréz Urbana de Manaus, AM. Mémoire de Master en sciences erbironnementales. Domaine Géotechnique, CCAMA. Manaus, AM, Brésil, 19g8, 200 p.

Cardoso F.B.E. - Propriedades a Comportamento Mecánico de Solos do Planálo Central Brasileiro. Tese de Doutorado em Geotecnia, Departamento de Engenharia Civl e Ambiental, FT, UnB, Brasi$1 \mathrm{ia}, \mathrm{DF}, 2002,358 \mathrm{p}$.

Fernandes Filho L.A., Costa M.L., Costa J.B.S. - Registros neotectôniques nos lateritos de Manaus. AM. Revue de Géosciences, Université de l'État: de São Paulo (UNNESP'), vol. 16, 1997

Inderbitzen A.L. - An erosion teșt for soils. Materials Research \& Standards $1(7)$, 1961, p. 553-554

Leroueil S.r Barbasa P.S.A. - Combined effect of fabric, bonding and partial saturation on yielding of soils. Proc. Asian Conf. on Unsaturated Solls, Singapure, 1999, p. 527-532.

Lima M.C. - Contribuiçāo Estudo do Processo Evoluţ́vo de Bocorocas na Área Urbana de Manaus. Mémoire de Master en Gébtechnique, Université de Brasilla, Brasília, DF, Brésil. 1999, $150 \mathrm{p}$.

L.ma M.C., Camapum de Garvallo J.. Frota C.A. - Caracterizaçăo Geotécnica do perf1] de solo tipico da cidade de Manaus. AM. Sols et Roches, revue latino-américaine de géotechnique, vol. 23 (3). 2000, p. $143-155$

Mortart D. Camapurn de Carvalho J. Modelo encaixado, uma proposição para a evoluçào das erosōes no Distrito Federal. 10 Congrès brésilien de mêcanique des sols et génie géotechnique, vol 4 ABMS, For do Iguacu, PR, Brésil, 1994. p. 1085-1092.

Sherand J.L. Dunnigan I.P., Decker R.S. Steele E.F - Pishole test for idertifying dispersive soils. Joumal of the Geotechnical Engineering Division, ASCE, 102 (1), 1976, p. 69-85.

Vilar O.M., Prandí E.C. - Erosia dos Solos. Sols dans l'intérieur de Säo Paulo leds. J.C.A. Cintra \& I.H. Albierol. ABMS, EESC, USP, Brésil, 1993, p. 177-206 